\title{
Green Synthesis, Characterization, and Application of Ascophyllum Nodosum Silver Nanoparticles
}

Sunil Kumar Mishra ( $\nabla$ skmishra.phe@itbhu.ac.in )

Banaras Hindu University Varanasi https://orcid.org/0000-0003-3749-5935

\section{Saket Sinha}

Banaras Hindu University Varanasi

\section{Amit Kumar Singh}

Banaras Hindu University Varanasi

\section{Prabhat Upadhyay}

National Institute of Immunology

\section{Diya Kalra}

Jamia Hamdard University

\section{Pradeep Kumar}

Banaras Hindu University Varanasi

Kavindra Nath Tiwari

Banaras Hindu University Varanasi

\section{Rajan Singh}

Banaras Hindu University Varanasi

Rakesh Kumar Singh

Banaras Hindu University Varanasi

\section{Arvind Kumar}

Banaras Hindu University Varanasi

\section{Alok Tripathi}

Banaras Hindu University Varanasi

\section{Research Article}

Keywords: Anti-oxidant, Silver nanoparticle, Anti-leishmanial activity, Cytotoxicity

Posted Date: September 29th, 2021

DOI: https://doi.org/10.21203/rs.3.rs-944036/v1

License: (c) (i) This work is licensed under a Creative Commons Attribution 4.0 International License. Read Full License 


\section{Abstract}

Emerging field of green nanotechnology has gained the attention of biologists due to the cost-effective and environment friendly advancements. The most preferred method being by biological means involving plants and their extracts, in the present investigation silver nanoparticles (AgNPs) were synthesised by sunlight-driven method, coated with aqueous extract (AE) of whole plant powder of Ascophyllum nodosum. High-resolution Scanning electron microscopy (HRSEM), Energy Dispersive X-ray (EDX), Highresolution Transmission electron microscopy (HRTEM) and particle size analysis were used to determine the nature of nanoparticles. The techniques confirmed the formation of spherical and particles of desired range of size. AgNPs exhibited a much greater DPPH (2,2-diphenyl-1-picryl-hydrazyl-hydrate) radical scavenging activity which was almost six to seven folds more than that exhibited by the AE alone. Antileishmanial and cytotoxic activity was evaluated on Leishmania donovani promastigote and amastigote. This raises the possibility of utilization of green synthesised AgNPs in leishmaniasis treatment.

\section{Introduction}

Nanotechnology involves the synthesis of particles with the size range up to $100 \mathrm{~nm}$. Research on nanoparticles has gained huge attention in the recent years due to its widespread utility in fields ranging from electronics to agriculture. The main characteristics responsible for their candidacy in large number of areas include their large surface area to surface volume ratio, small size, magnetic, optical, chemical and mechanical properties. The energy of the system is elevated due to an increase in the ratio of the atoms on surface to that on the bulk [1]. This attributes to increased anti-microbial properties in comparison with the bulk form. Metallic nanoparticles are a focus of interest due to their various therapeutic as well as bio-sensing abilities, chiefly their anti-microbial properties [2]. Nanoparticles in combination with noble metals such as silver, platinum, gold, copper and others are synthesised extensively as they possess catalytic, electrical, magnetic and optical properties [3]. AgNPs have proven to be the most efficient of all nano-sized metallic particles and are now considered to be a vital antibacterial agent. A pool of applications makes AgNPs the heavily utilized nanomaterial in pharmaceutical industry, optical sensors, domestic, and healthcare-related products, consumer products, diagnostics, medical device coatings, food industry and cosmetics [4]. Commercially, they are used nowadays in sporting equipment, where silver-embedded fabrics are used.

Various methods for production of the nanoparticles are reported in literature. Although the chemical method is till-date the most popular approach for the production of nanoparticles but it includes the use of toxic chemicals which makes the entire process hazardous as well as expensive. In case of AgNPs, there is till-date a need for cost-effective, commercially viable as well as environmentally clean route of synthesis for AgNPs [5]. For their high utility in the field of medicine, environmentally and biologically safe protocols need to be followed. This has led to development of procedures that today we call nontoxic 'green syntheses. Green here refers to the usage of plants or their extracts as reducing and stabilizing agents in place of poisonous toxic chemicals for biosynthesis of nano-sized particles [6]. 
AgNPs prepared using these biological methods have proven to exhibit high stability and yield along with increased solubility. This strategy demonstrates much promise over the other ones and is greatly biocompatible. Moreover, it can also be duly scaled up for the large-scale synthesis of nanoparticles. The photo-induced method produces particles of well-defined morphology and size under standardized conditions for translational research [7-9]. This hassle-free, rapid, economical, environment friendly approach has helped overcome the issue of use of nanoparticles in healthcare medications and in agriculture as biocides and fertilizers due to toxic components. In this method, the rate of the nanoparticle formation is directly proportional to the luminous intensity of the incident light. Many studies have been published on the sunlight induced route for the synthesis of AgNPs [10-12].

The brown seaweed Ascophyllum nodosum (Rock weed) is predominantly found on the rocky intertidal shores of the Atlantic region of Nova Scotia and New Brunswick, Canada. The species is confined to the North Atlantic region, which extends from the Arctic Circle to New Jersey in North America [13]. $A$. nodosum is considered an economically important seaweed, as it is harvested every year for its use in alginates (wound dressings), as a fertiliser and as an animal meal. Furthermore, evaluation of antioxidant property [14] and antimicrobial property [15] has been performed on the rockweed and significant results were reported [16]. Thus, we had selected the seaweed, A. nodosum for the first time to synthesise the AgNPs in a green eco-friendly way, through photo-induced method. The synthesised AgNPs were screened for their DPPH radical scavenging ability, parallel with the AE of $A$. nodosum. Anti-leishmanial activity was investigated on amastigote and promastigote cells for both AgNPs and AE.

\section{Material And Methods}

The whole plant powder of the seaweed $A$. nodosum was obtained from Canada, and is identified in the Department of Botany, Banaras Hindu University and authenticated for further study. Analytical grade Silver nitrate $\left(\mathrm{AgNO}_{3}\right)$, DPPH [1, 1 - diphenyl 2 - picryl hydrazyl], DMSO (Dimethyl sulfoxide) were obtained from Merck chemicals. The authentication of anti-leishmanial activity of AgNPs and AE was evaluated on cloned line of Leishmania donovani promastigotes. Mueller Hinton agar and broth media (Himedia Lab Ltd. Mumbai) were used for studying the growth activity in the culture. All the glassware was washed with distilled water and dried properly.

\section{Photo-induced synthesis of Silver Nanoparticles (AgNPs)}

A reaction mixture was prepared by taking $0.1 \mathrm{ml} \mathrm{AE}$ with $10 \mathrm{ml}$ of $1 \mathrm{mM} \mathrm{AgNO}_{3}$ solution. The concentration of the AE was maintained at $1 \mathrm{mg} / \mathrm{ml}$. The biosynthesis of nanoparticles was done by exposing the reaction mixture to the sunlight for a duration of 5-60 minutes at 5 minutes' interval. The intensity of the incident solar light was found as $63000 \mathrm{~lx}$. Meanwhile, the UV-absorbance was also measured with respect to time at 200 to $800 \mathrm{~nm}$ using the Shimadzu UV-1800 instrument.

\section{Effect of varying concentrations of $\mathrm{AgNO}_{3}$}


The effect of different concentrations of $\mathrm{AgNO}_{3}$ on the formation of nanoparticles was also studied. The concentration of $\mathrm{AgNO}_{3}$ was made to vary from $0.5 \mathrm{mM}$ to $3.0 \mathrm{mM}$ with an increasing difference of 0.5 $\mathrm{mM}$. Whereas, the $A E$ concentration was kept constant $(1 \mathrm{mg} / \mathrm{ml})$ in each reaction mixture. Various reaction mixtures of multiple $\mathrm{AgNO}_{3}$ concentrations with $\mathrm{AE}$ were exposed to sunlight for about 5 minutes. By this way, the effect of altered concentrations of $\mathrm{AgNO}_{3}$ for the AgNPs synthesis is studied by the UV-visible absorption spectroscopy.

\section{Effect of the Aqueous extract (AE) inoculum dose}

Similarly, the effect of different quantities of $A E$ on the synthesis of nanoparticles was studied by keeping the concentration of $\mathrm{AgNO}_{3}$ constant $(1 \mathrm{mM})$. The initial amount of $\mathrm{AE}$ was standardized by altering its volume from $200 \mu \mathrm{l}$ to $2000 \mu \mathrm{l}$, for a common exposure of 5 minutes in the sunlight. The optimum dose of the AE was confirmed by following the same procedure of nanosynthesis.

\section{Characterization of the green synthesised AgNPs}

Optical properties of the biocompatible nanotized silver particles were evaluated using the UV-Visible spectroscopy (Shimadzu UV-1800). High-resolution Scanning electron microscopy (HRSEM) (Nova Nano SEM450) was performed to analyse the morphology of the nanoparticles at accelerating voltage of $10 \mathrm{kV}$. The beam current was kept at $1 \mathrm{nA}$, with average measuring time of 7 seconds. The elemental composition was confirmed meanwhile, with the Energy Dispersive X-ray (EDX) detector, which was equipped with the HRSEM. The High-resolution Transmission electron microscopy (HRTEM; TECHNI G2 20 TWIN; FEI) at an accelerating voltage of $200 \mathrm{kV}$, was used in studying the size and morphology of AgNPs. 'Image J' software was used to calculate the average particle size. Particle size analysis of biosynthesized AgNPs was obtained using Dynamic Light Scattering Malvern-Zeta sizer (Particulate systems, Nanoplus, Shimadzu).

\section{DPPH radical scavenging assay}

This study was performed to find out the antioxidant potential of the synthesized AgNPs, with respect to the original AE. Hence DPPH radical scavenging activity of both, AE of A. nodosum, and the green synthesized AgNPs was studied and the results were compared. DPPH solution of $0.004 \%$ was prepared in distilled water. $2.96 \mathrm{ml}$ of DPPH solution was mixed with various concentrations of samples $(50 \mu \mathrm{g} / \mathrm{ml}$ $700 \mu \mathrm{g} / \mathrm{ml}$ ). The solution was then kept at room temperature in dark condition for 20 minutes [17]. The absorbance of the reaction mixture was checked after 20 minutes with UV-Vis spectrophotometer at a wavelength of $517 \mathrm{~nm}$, where DPPH was used as a control. The following equation was used for the calculation of percentage inhibition:

$\left[\left(\right.\right.$ Absorbance $_{\text {control-Absorbance }}$ sample $) /\left(\right.$ Absorbance $\left.\left._{\text {control }}\right)\right] \times 100$

The mean and standard deviation (SD) were calculated by repeating all the experiments thrice. P values of $\leq 0.001$ were considered significant. The half maximal Inhibitory Concentration $\left(\mathrm{IC}_{50}\right)$ was calculated 
by comparing with that of standard (ascorbic acid is taken as standard), by linear regression analysis.

\section{Anti-leishmanial activity}

\section{Culture conditions}

L. donovani promastigote parasites were grown in vitro in DMEM (Invitrogen, USA) supplemented with 10 $\%$ FBS and antibiotics (Streptomycin $100 \mu \mathrm{g} / \mathrm{mL}$, penicillin $100 \mathrm{U} / \mathrm{ml}$, gentamycin $20 \mu \mathrm{g} / \mathrm{ml}$, Sigma, USA) at $\mathrm{pH} 7.2$ and finally incubated in Bio-Oxygen Demand (BOD) incubator at $26^{\circ} \mathrm{C}$. Mouse macrophages, RAW 264.7 were cultured and allowed to adhere in the 24 well plate at a concentration of $5 \times 10^{5}$ cells $/ 100 \mu \mathrm{L} /$ well in DMEM medium having $10 \% \mathrm{FBS}, 1 \%$ streptomycin, penicillin and incubated at $37^{\circ} \mathrm{C}$ in $5 \% \mathrm{CO}_{2}$ incubator.

\section{Determination of effects of AgNPs on proliferation, infectivity and cell viability of $L$. donovani}

Macrophages (RAW 264.7) were infected by flagellated promastigote form of $L$. donovani. Post-infection, promastigotes were phagocytosed and transformed into non-motile amastigotes form of $L$. donovani. Culture plate was incubated for 4-6 hours, after completion of incubation, culture plate was given onetime wash. Furthermore, the test compounds $(200 \mu \mathrm{L})$ were added two fold serially diluted over 8 concentrations ranging from $100 \mu \mathrm{g} / \mathrm{mL}$ to $0.78 \mu \mathrm{g} / \mathrm{mL}$ and then incubated for 48 hours. After 48 hours, bio-evaluation of anti-leishmanial activity towards intracellular amastigotes was measured by using direct counting assays following fixation and Giemsa staining. All assays were performed in triplicates and drug activity was analysed under phase contrast microscopy.

Promastigotes in their logarithmic phase were seeded in a 96 well culture plate $\left(1 \times 10^{6}\right.$ cells $100 \mu \mathrm{L} /$ well) for identifying $\mathrm{IC}_{50}$ of synthesised particles. The plate was seeded without (control wells) or with $100 \mu \mathrm{L}$ of reaction mixture per well such that the final concentration of samples in the well varied from 100 $\mu \mathrm{g} / \mathrm{mL}$ to $0.78 \mu \mathrm{g} / \mathrm{mL}$ (serial dilution) and incubated for 48 hours. Furthermore, to determine the inhibition percentage of respective test compound MTT (3-(4, 5-dimethylthiazolyl-2)-2, 5diphenyltetrazolium bromide) assay was done. This, MTT assay was used to check the presence of viable cells, after the treatment of test compound. Mechanism behind this assay is the conversion of MTT to formazan by mitochondrial enzymes served as indicator of cell viability and the amount of formazan produced indicates the viable cells with active metabolism. Briefly MTT ( $5 \mathrm{mg} / \mathrm{mL}, 30 \mu \mathrm{L} /$ well) was added to each well and further plates were allowed to incubate for 4 hours at $37^{\circ} \mathrm{C}$. Subsequently after $4 \mathrm{hr}$ plates were centrifuged at $2500 \mathrm{rpm}$ for 5 minutes, the supernatant was discarded and DMSO (100 $\mathrm{LL} /$ well) was added to solubilize the precipitated formazan. Accordingly, cell viability was measured at $540 \mathrm{~nm}$ using Elisa plate reader. All experiments were repeated thrice with each drug being used in triplicates.

The parasite growth inhibition is determined by comparing the activity of control wells (normal activity) with that of treated wells (inhibited activity) via the equation 
$\%$ Inhibition $=$ OD of control well - OD of treated well* $100 / 0 D$ of control well

All results were represented as $\mathrm{IC}_{50} \pm \mathrm{SD}$ and were calculated by linear interpolation as given below:

$\log (\mathrm{IC} 50)=\log (\mathrm{X} 1)+(50-\mathrm{Y} 1) /(\mathrm{Y} 2-\mathrm{Y} 1)[\log (\mathrm{X} 2)-\log (\mathrm{X} 1)]$

where $\mathrm{X} 1$ = drug concentration giving \% parasitic inhibition, $\mathrm{X} 1>50 \%, \mathrm{X} 2=$ drug concentration giving \% parasitic inhibition, $\mathrm{Y} 2<50 \% ; \mathrm{Y} 1$ and $\mathrm{Y} 2$ are respective cell densities (control and test).

\section{Cytotoxicity assay (Anti-Leishmanial)}

For evaluation of cytotoxicity of test compounds against mice macrophages cell line, RAW 264.7 were cultured in DMEM containing $10 \% \mathrm{FBS}$ at $37^{\circ} \mathrm{C}$ in $5 \% \mathrm{CO}_{2}$ atmosphere. After that, the macrophages were seeded in a 96 well micro titer plate at a concentration of $1 \times 10_{6}$ cells $/ \mathrm{ml}$ in the presence of compounds which were two fold serially diluted over 8 concentration points ranging from $100 \mu \mathrm{g}$ so that the final concentration of compounds became range from $50 \mu \mathrm{g} / \mathrm{ml}$ to $0.39 \mu \mathrm{g} / \mathrm{ml}$ and incubated at $37^{\circ} \mathrm{C}$ in $5 \%$ $\mathrm{CO}_{2}$ atmosphere. For standard control, Miltefosine which is a commercially available anti-leishmanial drug was used. Cells which were not treated with any drug taken as control. Further the S.I. was calculated by ratio between $\mathrm{CC}_{50}$ and $\mathrm{IC}_{50}$ of macrophage amastigote.

\section{Results}

\section{Photo Induced synthesis of Silver Nanoparticles (AgNPs)}

An observation about the colour of the reaction mixture was made that it became darker with the increase in exposure time. There was a change in the physical appearance of the solution, which was marked by the rise in the intensity of the solution's colour to deep red brown colour. There was a continuous rise in the UV-absorbance of the reaction mixture at around $415 \mathrm{~nm}$, with respect to time. Also, the maxima of the peak observed for absorbance of reaction mixture to exposed sunlight for different durations, showed a blue shift. This showcases the biosynthesis of AgNPs with passage of time. The intensity of the Surface Plasmon Resonance (SPR) band elevated up to 15 minutes, after that, it ceased to increase. Whereas further no change in the intensity of SPR band was observed, indicating the termination of the synthesis process. Conclusively, 10 minutes was marked as the optimum time of exposure to sunlight in the photo-induced synthesis of AgNPs (Figure 1A).

\section{Effect of varying concentrations of $\mathrm{AgNO}_{3}$}

The colour of the reaction mixture became darker with continuing increase in $\mathrm{AgNO}_{3}$ concentration at the fixed reaction time. Our observation showed that, when $\mathrm{AgNO}_{3}$ concentration increases, the peak maxima also increase correspondingly (Figure 1B). The spectra showed that the concentration of $3 \mathrm{mM}$ showed good SPR band, indicating proper formation of nanoparticles. 
The findings stated that while increasing the AE dose, the sharpness and intensity of the SPR band continued to increase up to $1000 \mu \mathrm{l}$ and later stopped. $200 \mu \mathrm{l}$ to $1000 \mu \mathrm{l}$ of AE formed a shoulder peak and increased intensity towards blue region. This signifies that the formation of nanoparticles increased with increase in AE inoculum dose up to $1000 \mu \mathrm{l}$ (Figure 1C). Thus, $1000 \mu \mathrm{l}$ was considered as the optimum dose of the AE for nanosynthesis.

\section{Characterization of the green synthesised AgNPs}

\section{HRSEM and EDX analysis}

Spherical AgNPs were synthesised as confirmed by High Resolution-Scanning electron microscopy and the size ranged between $36 \mathrm{~nm}$ to $69 \mathrm{~nm}$ (Figure 2A). Some big sized nanoparticles were seen, which could be because of the accumulation or overlapping of the particles. Synthesis of AgNPs was evidenced by the EDX spectra that were determined between $2 \mathrm{keV}$ to $4 \mathrm{keV}$, which evidently displayed a strong spectral signal in silver region at $3.5 \mathrm{keV}$ (Figure 2B). In EDX spectra, presence of signals of carbon, oxygen and sodium indicated the presence of bioactive compounds of the AE. This result supports the presence of $A E$ as capping agent with the nanoparticles.

\section{High Resolution-Transmission electron microscopy (HRTEM) analysis}

HRTEM images of the AgNPs were shown in the Figure 3A and B. Nanoparticles with spherical shape and smooth surface prove to be very stable. Analysis of the selected area electron diffraction (SAED) pattern as shown in Figure $3 \mathrm{C}$ confirms the crystalline nature of the AgNPs. The particle size distribution of AgNPs was between $14 \mathrm{~nm}$ to $30 \mathrm{~nm}$, with an average particle size of $27 \mathrm{~nm}$.

\section{Particle size analysis}

A dynamic light scattering method was adopted to analyse the particle sizes to give a picture of particles size distribution of the synthesized AgNPs (Figure 4). Particles of various sizes ranging from $55.1 \mathrm{~nm}$ to $126.7 \mathrm{~nm}$ were observed. The average particles size for AgNPs was found to be $164.6 \mathrm{~nm}$. Particle size analyser showed the occurrence of AgNPs with low polydispersity index, PDI (0.206). The average particle size and PDI exhibited that the produced AgNPs are mono dispersed.

\section{DPPH (2,2-diphenyl-1-picryl-hydrazyl-hydrate) radical scavenging assay}

The percentage inhibition values for DPPH radical scavenging activity of both AE and AgNPs are shown in the Table 1. The AgNPs $\left(\mathrm{IC}_{50}=103.79 \mu \mathrm{g} / \mathrm{ml}\right)$ exhibited far greater free radical scavenging ability, in comparison with the $\mathrm{AE}\left(\mathrm{IC}_{50}=765.92 \mu \mathrm{g} / \mathrm{ml}\right)$.

\section{Anti-leishmanial activity}

Effects of AgNPs on proliferation of $L$. donovani promastigotes 
Proliferation of $L$. donovani promastigotes was investigated in cases of AE and AgNPs treatment and also compared with anti-leishmanial drug, Miltefosine. The promastigotes in the control group not exposed to any treatment appeared to have intact normal cell morphology along with showing active proliferation. However, cell density in cells treated with AE, AgNPs or miltefosine significantly decreases (Figure 5).

\section{Effect of AgNPs on infectivity of $L$. donovani amastigotes}

Another biological aspect of infectivity of Leishmania parasites, being the major cause of Leishmaniasis was evaluated. Macrophages infected with $L$. Donovan and the in vitro anti-amastigote activity of AgNPs and $A E$ was analysed. Active infection of macrophages by L. donovani promastigotes in control cells was seen The promastigotes actively transformed into amastigotes. Macroscopic images of $L$. donovani promastigotes exposed to AE infected the macrophages less efficiently than they did in the control group. Further decrease in infection of promastigotes in macrophages is observed in AgNPs treated group as compared to Miltefosine treated group (Figure 6). These results indicate that infectivity ratios of $L$. donovani amastigotes reduce when treated with $A E$ but complete inhibition of infection is observed in AgNPs treated group.

\section{Percent cell viability on $L$. donovani promastigotes}

$\mathrm{AE}$ and AgNPs both displayed irregular cell growth but AgNPs are perceived to be more effective. Toxicity was directly proportional to the dose as we observed more toxicity at a higher dose (200) which was not the case at lower dose. Thus, AgNPs at lower dose show promising results in terms of being less toxic and safe (Figure 7).

\section{Cytotoxicity Assay (Anti-leishmanial)}

$I_{50}$ values of $A E$ and AgNPs were calculated on promastigote forms of $L$. donovani as well as $L$. donovani intra-macrophage amastigotes forms. It was higher on amastigotes $(52.48 \mu \mathrm{M})$ as compared to on promastigote forms $(42.01 \mu \mathrm{M})$ as shown in Table 2. The $I_{50}$ values for AgNPs were interestingly similar on both promastigote and amastigote forms and much comparable to the standard drug, miltefosine that we used. Further we estimated the ratio of toxic concentration of an AE and AgNPs against its effective bioactive concentration on intra-macrophage amastigotes forms, also known as the Selectivity Index (SI). The window between the cytotoxicity and anti-leishmanial activity depends on the SI value and is directly proportional to its efficacy in vivo. The SI value of the green synthesised AgNPs was maximum and almost three-fold higher than miltefosine. This proves the potent anti-leishmanial activity of our fabricated AgNPs.

\section{Discussion}

The formation of AgNPs in the sunlight was confirmed by the physical alteration in the colour of the reaction mixture. Intensity of the coloured solution increases with passing time due to the reduction of the 
Ag ion. The phytochemicals of $A E$ used is responsible for the capping of the ion to prevent aggregation. This fundamental phenomenon behind the colour change is SPR, which occurs due to the oscillations of the free electrons[18]. Additionally, the SPR effect is dependent on the nature, shape and size of the nanoparticles [19]. The UV-Visible spectrophotometer, used for spectral characterization of the nano-sized particles, identifies the intensity of SPR band by measuring the SPR absorbance. The AgNPs exhibited a characteristic SPR band, where the $\lambda$ max values ranges from $400 \mathrm{~nm}-500 \mathrm{~nm}$ [20]. We obtained the peak intensity of the SPR band at $415 \mathrm{~nm}$ (Figure 1), which indicated a characteristic SPR of the AgNPs.

The characterization of AgNPs in this study also obey the Mie's theory which says that presence of a single SPR band indicates the formation of spherical AgNPs. Observation of two or more SPR bands, shows variation in shapes of AgNPs [21]. A uniform population of spherical AgNPs as indicated by one band only, were successful synthesised. This was further supported by the HR-TEM and HR-SEM images depicting spherical and stable particles (Figure 2A and $3 \mathrm{~A}$ ).

Sunlight-driven protocol of nanoparticles occurs because of the superoxide from photoirradiation which converts Ag to AgNPs. The time of exposure of sunlight to the reaction mixture for the synthesis of desirable nanoparticles also plays a vital role. It was noted that up to 15 minutes, the intensity of the SPR band increased, which is a clear evidence of the fact that the process of formation of the particles does not go beyond a time duration of 15 minutes and eventually terminates. A SPR band of maximum intensity at 15 minutes indicated the synthesis process of AgNPs due to reduction of $\mathrm{Ag}^{+}$ions to $\mathrm{Ag}^{0}$. Beyond which the intensity saturated depicting stopping of synthesis process.

The two main components for the synthesis of these nano-sized particles includes the $\mathrm{AgNO}_{3}$ and the $\mathrm{AE}$ of plant that was used. The quality of nanoparticles synthesised is dependent on the concentration of these two constituents. The size to distribution ratio of the nanoparticle, referred to as the Polydispersity Index (PDI) is an important factor for determining uniformity of nanoparticles. Mie's theory further stated that the chances of production of polydispersed AgNPs increases with higher concentration of the inoculum, and even the SPR band may get split [21]. Standardisation of the procedure was necessary, so optimization of the dose of inoculum was carried out. Reaction mixture with $1000 \mu \mathrm{l}$ of AE showed maximum absorbance. At dose higher than $1000 \mu \mathrm{l}$, formation of non-uniform population of particles took place which showed band with low intensity. In similar manner, the concentration of $\mathrm{AgNO}_{3}$ was also optimized. An intensity maximum was obtained with $3 \mathrm{mM}$ concentration of $\mathrm{AgNO}_{3}$. Hence $3 \mathrm{mM}$ was found to be the appropriate concentration of $\mathrm{AgNO}_{3}$ for the synthesis of AgNPs.

The particle size is analysed by Dynamic light scattering method which depicts the particles size distribution of the synthesized AgNPs by the AE. The range $55.1 \mathrm{~nm}$ to $126.7 \mathrm{~nm}$ of particle size distribution of AgNPs indicated the presence of various size of the particles. Average particles size for AgNPs was $164.6 \mathrm{~nm}$ and PDI showed that the produced AgNPs were monodispersed.

Vijay kumar et al. hypothesised the mechanism behind the synthesis of nanoparticles under sunlight [22]. The photo energy from the sun is absorbed by the polyphenolic rich AE and gets excited to a higher state 
by substituting its $\mathrm{O}-\mathrm{H}$ groups with $\mathrm{Ag}^{+}$ions. The monovalent cation $\mathrm{Ag}^{+}$ions get reduced to $\mathrm{Ag}^{0}$ leading to its nucleation and ultimately results in the formation of nanoclusters. The stability of the AgNPs is achieved due to the polyphenolic protein in the AE that act as a capping agent [21]. This capping activity of the AE was verified by spectral signal at $3.5 \mathrm{keV}$ in obtained EDX spectra (Figure 2B) of AgNPs that showed the presence of elements of the AE, along with the AgNPs. With this, a successful method of preparation of stable AgNPs using A. nodosumas a capping agent is reported. Thus, the AE coated AgNPs are advantageous than physical and chemical methods producing stable and spherical nanoparticles in an eco-friendly way eliminating the use of toxic chemicals.

The natural antioxidants have prophylactic and therapeutic effects for combating many diseases like cancer, cardiovascular disease and several age-related diseases [23]. DPPH assay is an ideal and easy method for the evaluation of antioxidant ability. This method is commonly used to evaluate the radical scavenging ability in vegetables, brans, linoleic acids and herbs [24]. An antioxidant reduces or in other term, scavenges the DPPH free radical. The AgNPs synthesised exhibited satisfactory free radical scavenging ability, showing better efficacy than the AE. The $\mathrm{IC}_{50}$ value of the AgNPs was somewhat nearer to the standard value which adds on to is antioxidant potential. Various other methods can be adopted further prove its properties like superoxide radical scavenging, lipid per oxidation activity and reducing power ability with subsequent in vivo studies.

Potent anti-leishmanial activity was witnessed in both, AE and AgNPs. Dose dependent activity was found in promastigote as well as amastigote. $\mathrm{IC}_{50}$ in both $\mathrm{AE}$ and AgNPs was 42.01 and 5.86 respectively and were found effective in promastigote as compared to the standard (7.2). A similar trend of 52.48, $5.89,8.1$ was observed in amastigote for AE, AgNPs and standard respectively.

\section{Conclusion}

Plant extract mediated synthesis of nanoparticles has definitely filled the void of the need for a safer method for our ecosystem. The researchers of the medical field find utmost potential in these nanoparticles being utilised for drug delivery and other healthcare products. In this study, AE of $A$. nodosumas served as a capping and stabilising agent for photo-induced synthesis of AgNPs of 30-60 nm in size. The micro structural analysis revealed the crystalline nature of the particles. The synthesised AgNPs showed remarkable DPPH radical scavenging ability owing to its antioxidant properties. Antileishmanial activity was exceptionally viable in both AE and AgNPs. A low dose of AE and AgNPs is considered safe for use. These findings all together support the tendency of Ascophyllum nodosum to efficiently synthesise AgNPs which could be utilized for its anti-leishmanial properties.

\section{Declarations}

\section{Competing interests}

The authors declare that they have no competing interests. 
Availability of data and materials

All data sets analyzed and attached with a manuscript in the form of table and figure.

\section{Ethics approval and consent to participate}

No animal or human studies were carried out by the authors for this article.

\section{Consent for publication:}

On behalf of all author, the corresponding author give his full consent to the journal for publication of the manuscript.

\section{Authors' contributions}

SS and AKS synthesized the nano particle and characterization and analysis done with PK. SKM and KNT conceptualized the experiment for study and helped for resources. RS, AK performed the cell line experiments and resources available by RKS and AT. PU and DK contributed in the manuscript writing and data analysis. All authors read and approved the final manuscript.

\section{Funding}

No funding was received

\section{Conflict of interest}

No conflict of interest in this work.

\section{Disclosure}

The author reports no conflicts of interest in this work.

\section{Acknowledgements}

We are thankful to the staff of Central Instrument facility, Indian Institute of Technology, BHU and Department of Botany, Banaras Hindu University Varanasi.

\section{References}

1. Dwivedi AD, Gopal K (2010) Biosynthesis of silver and gold nanoparticles using Chenopodium album leaf extract. Colloids Surfaces A Physicochem Eng Asp 369:27-33.

https://doi.org/10.1016/j.colsurfa.2010.07.020

2. Sondi I, Salopek-Sondi B (2004) Silver nanoparticles as antimicrobial agent: A case study on E. coli as a model for Gram-negative bacteria. J Colloid Interface Sci 275:177-182.

https://doi.org/10.1016/j.jcis.2004.02.012 
3. Bar H, Bhui DK, Sahoo GP, et al (2009) Green synthesis of silver nanoparticles using latex of Jatropha curcas. Colloids Surfaces A Physicochem Eng Asp 339:134-139.

https://doi.org/10.1016/j.colsurfa.2009.02.008

4. Xu L, Wang YY, Huang J, et al (2020) Silver nanoparticles: Synthesis, medical applications and biosafety. Theranostics 10:8996-9031

5. Singh J, Dutta T, Kim KH, et al (2018) "Green" synthesis of metals and their oxide nanoparticles: Applications for environmental remediation. J. Nanobiotechnology 16:84

6. Upadhyay P, Mishra SK, Purohit S, et al (2019) Antioxidant, antimicrobial and cytotoxic potential of silver nanoparticles synthesized using flavonoid rich alcoholic leaves extract of Reinwardtia indica. Drug Chem Toxicol 42:65-75. https://doi.org/10.1080/01480545.2018.1488859

7. Upadhyay P, Mishra SK, Purohit S, et al (2019) Antioxidant, antimicrobial and cytotoxic potential of silver nanoparticles synthesized using flavonoid rich alcoholic leaves extract of Reinwardtia indica. Drug Chem Toxicol. https://doi.org/10.1080/01480545.2018.1488859

8. Mishra AK, Tiwari KN, Saini R, et al (2020) Green Synthesis of Silver Nanoparticles from Leaf Extract of Nyctanthes arbor-tristis L. and Assessment of Its Antioxidant, Antimicrobial Response. J Inorg Organomet Polym Mater 30:2266-2278. https://doi.org/10.1007/s10904-019-01392-w

9. Padalia H, Moteriya P, Chanda S (2015) Green synthesis of silver nanoparticles from marigold flower and its synergistic antimicrobial potential. Arab J Chem 8:732-741

10. Wang X, Tang H, Tian X, et al (2020) Sunlight and UV driven synthesis of Ag nanoparticles for fluorometric and colorimetric dual-mode sensing of ClO. Spectrochim Acta - Part A Mol Biomol Spectrosc 229:117996. https://doi.org/10.1016/j.saa.2019.117996

11. Nadal E, Barros N, Peres L, et al (2020) In situ synthesis of gold nanoparticles in polymer films under concentrated sunlight: Control of nanoparticle size and shape with solar flux. React Chem Eng 5:330341. https://doi.org/10.1039/c9re00439d

12. Singh C, Kumar J, Kumar P, et al (2019) Green synthesis of silver nanoparticles using aqueous leaf extract of Premna integrifolia (L.) rich in polyphenols and evaluation of their antioxidant, antibacterial and cytotoxic activity. Biotechnol Biotechnol Equip 33:359-371.

https://doi.org/10.1080/13102818.2019.1577699

13. Pereira L, Morrison L, Shukla PS, Critchley AT (2020) A concise review of the brown macroalga Ascophyllum nodosum (Linnaeus) Le Jolis. J. Appl. Phycol. 32:3561-3584

14. Liu X, Luo G, Wang L, Yuan W (2019) Optimization of antioxidant extraction from edible brown algae Ascophyllum nodosum using response surface methodology. Food Bioprod Process 114:205-215. https://doi.org/10.1016/j.fbp.2019.01.003

Page $12 / 20$ 
15. Dell'Anno M, Sotira S, Rebucci R, et al (2020) In vitro evaluation of antimicrobial and antioxidant activities of algal extracts. Ital J Anim Sci 19:103-113.

https://doi.org/10.1080/1828051X.2019.1703563

16. Ertani A, Francioso O, Tinti A, et al (2018) Evaluation of Seaweed Extracts From Laminaria and Ascophyllum nodosum spp. as Biostimulants in Zea mays L. Using a Combination of Chemical, Biochemical and Morphological Approaches. Front Plant Sci 9:428.

https://doi.org/10.3389/fpls.2018.00428

17. Rawat V, Sharma A, Bhatt VP, et al (2019) Sunlight mediated green synthesis of silver nanoparticles using Polygonatum graminifolium leaf extract and their antibacterial activity. In: Materials Today: Proceedings. Elsevier Ltd, pp 911-916

18. Amendola V, Pilot R, Frasconi M, et al (2017) Surface plasmon resonance in gold nanoparticles: A review. J. Phys. Condens. Matter 29:203002

19. Jain PK, Huang X, El-Sayed IH, El-Sayed MA (2007) Review of some interesting surface plasmon resonance-enhanced properties of noble metal nanoparticles and their applications to biosystems. Plasmonics 2:107-118. https://doi.org/10.1007/s11468-007-9031-1

20. Sastry M, Mayya KS, Bandyopadhyay K (1997) pH Dependent changes in the optical properties of carboxylic acid derivatized silver colloidal particles. Colloids Surfaces A Physicochem Eng Asp 127:221228. https://doi.org/10.1016/S0927-7757(97)00087-3

21. Mie G, Mie, G. (1976) Contributions to the optics of turbid media, particularly of colloidal metal solutions. cotm 25:377-445

22. Kumar V, Bano D, Mohan S, et al (2016) Sunlight-induced green synthesis of silver nanoparticles using aqueous leaf extract of Polyalthia longifolia and its antioxidant activity. Mater. Lett. 181:371-377

23. Liu Z, Ren Z, Zhang J, et al (2018) Role of ROS and nutritional antioxidants in human diseases. Front. Physiol. 9:477

24. Cheng Z, Moore J, Yu L (2006) High-throughput relative DPPH radical scavenging capacity assay. J Agric Food Chem 54:7429-7436. https://doi.org/10.1021/jf0611668

\section{Tables}

Table 1. DPPH Radical scavenging assay of aqueous extract and Silver nanoparticles. 


\begin{tabular}{|c|c|c|}
\hline $\begin{array}{c}\text { Concentration } \\
(\boldsymbol{\mu} \mathrm{g} / \mathrm{m})\end{array}$ & $\begin{array}{c}\text { Percentage Inhibition of AE } \\
(\mathrm{mean} \pm \mathrm{S} . \mathrm{E})\end{array}$ & $\begin{array}{c}\text { Percentage Inhibition of AgNPs } \\
(\mathrm{mean} \pm \mathrm{S} . \mathrm{E})\end{array}$ \\
\hline 50 & $10.044 \pm 0.47^{* *}$ & $2.674 \pm 0.72^{* *}$ \\
\hline 100 & $14.032 \pm 0.99^{* *}$ & $8.024 \pm 0.52^{* *}$ \\
\hline 200 & $25.892 \pm 1.15^{* *}$ & $6.872 \pm 1.54^{* *}$ \\
\hline 400 & $26.770 \pm 1.32^{* *}$ & $21.193 \pm 1.33^{* *}$ \\
\hline 600 & $64.060 \pm 1.01^{* *}$ & $30.041 \pm 1.91^{* *}$ \\
\hline 800 & $72.990 \pm 1.10^{* *}$ & $36.213 \pm 1.89^{* *}$ \\
\hline 1000 & $77.232 \pm 1.33^{* *}$ & $51.028 \pm 1.21^{* *}$ \\
\hline $\mathrm{IC}{ }_{50}$ & $765.92 \mu \mathrm{g} / \mathrm{ml}$ & $103.73 \mu \mathrm{g} / \mathrm{ml}$ \\
\hline
\end{tabular}

$\mathrm{IC}_{50}$ value of Ascorbic acid $=89.10 . * *$ Significant at $\mathrm{P}$ value $<0.001$.

Table 2. S.I. of intra-macrophage amastigotes calculated by ratio between $\mathrm{CC}_{50}$ and $\mathrm{IC}_{50}$ of macrophage amastigote forms

\begin{tabular}{l|l|l|l|l|l|}
\hline ound & $\begin{array}{l}\text { Mol. } \\
\text { wt. }\end{array}$ & $\begin{array}{l}\mathrm{IC}_{50}(\mu \mathrm{M}) \text { on } \\
\text { promastigote } \\
\text { forms of } L . \\
\text { donovani }\end{array}$ & $\begin{array}{l}\mathrm{IC}_{50}(\mu \mathrm{M}) \text { on } \\
\begin{array}{l}L . \\
\text { donovani intra- } \\
\text { macrophage } \\
\text { amastigotes } \\
\text { forms }\end{array}\end{array}$ & $\begin{array}{l}\mathrm{CC}_{50}(\mu \mathrm{M}) \\
\text { on RAW } \\
264.7 \\
\text { macrophage }\end{array}$ & $\begin{array}{l}\mathrm{SI}_{\left(\mathrm{CC}_{50} / \mathrm{IC}_{50}\right.} \\
\text { of intra- } \\
\text { macrophage } \\
\text { amastigotes } \\
\text { forms })\end{array}$ \\
\hline $\mathrm{S}$ & & 52.48 & $<200$ & 3.67 \\
\hline $\mathrm{t}$ & & 5.86 & 5.89 & $<200$ & 33.95 \\
\hline articles & $\begin{array}{l}407.568 \\
\mathrm{~g} / \mathrm{mol}\end{array}$ & 7.2 & 8.1 & $<100$ & 12.34 \\
\hline
\end{tabular}

\section{Figures}




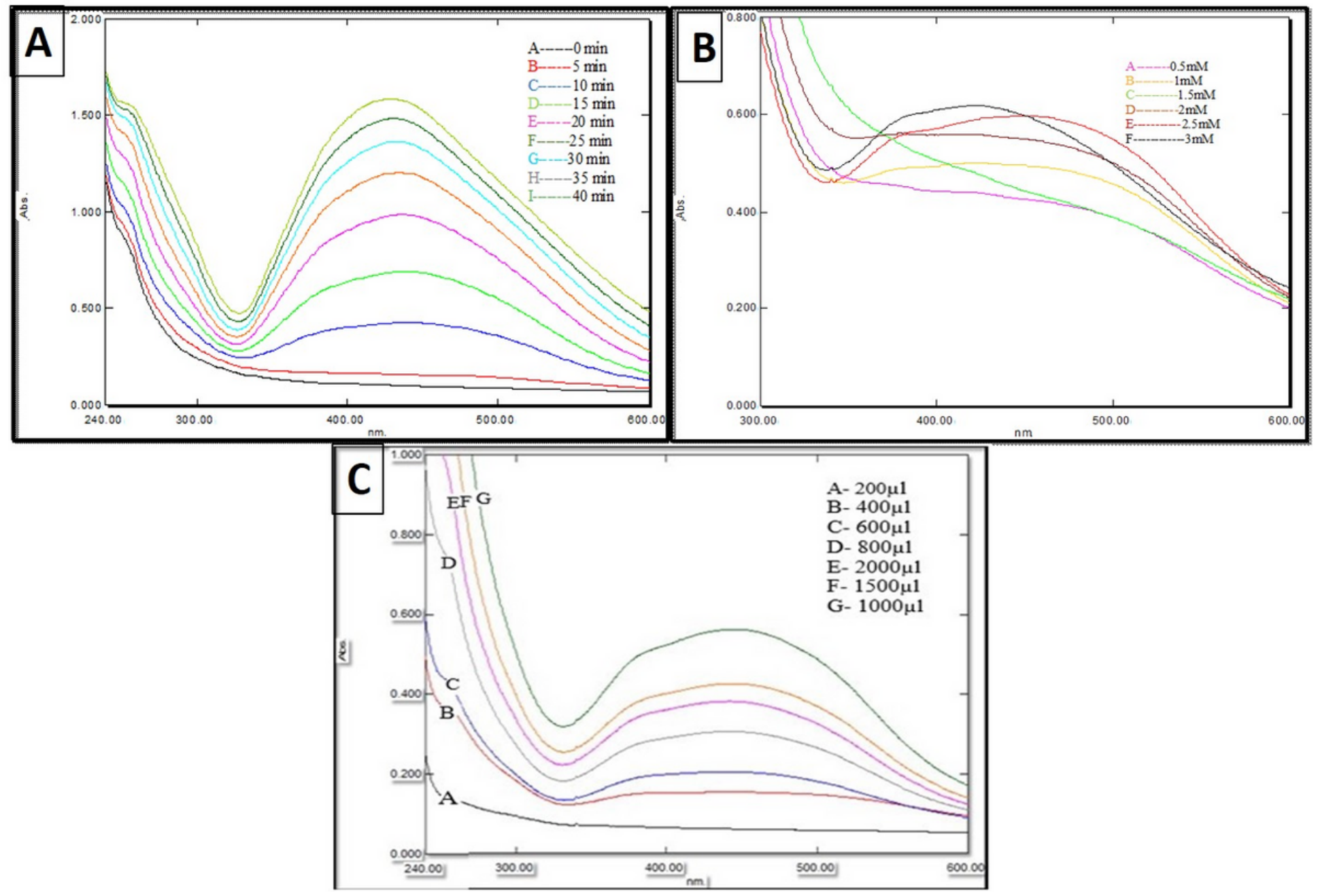

Figure 1

Green synthesis of silver nanoparticles (AgNPs) using Ascophyllum nodosum aqueous extract by Photoinduced method. (A) UV absorbance of the reaction mixture with respect to time duration of sunlight exposure. The Curve-D (15 minutes) showed the maximum absorbance. (B) Green synthesis of AgNPs at different concentration of AgNO3. The Curve-F (3mM) showed maximum absorbance. (C) Green synthesis of AgNPs at different inoculum dose of aqueous extract. Curve-G $(1000 \mu \mathrm{l})$ showed the maximum absorption. 


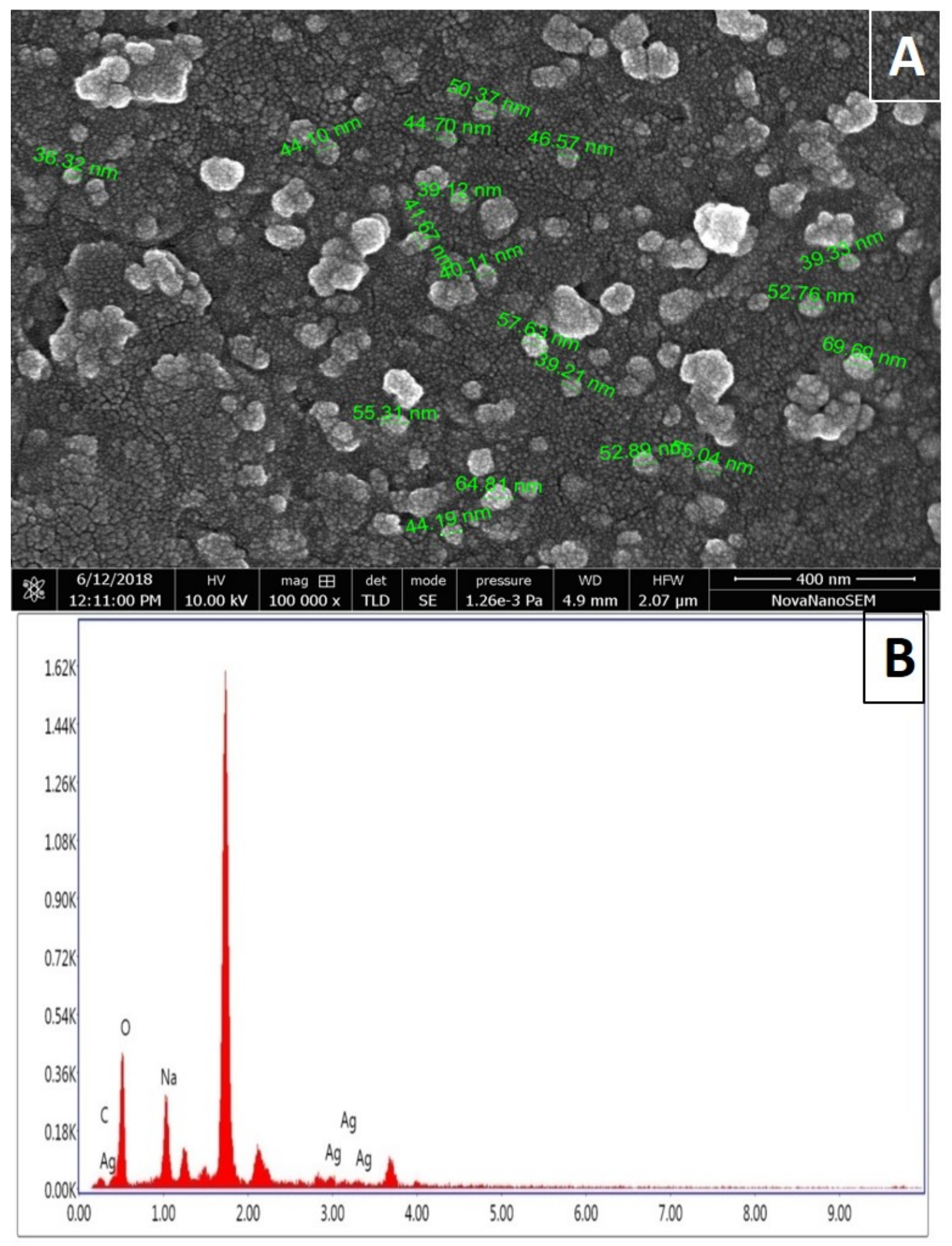

Lsec; 30.00 Cnts 0.000 keV Det: Octane Plus Det

\section{Figure 2}

Characterization of the green synthesised silver nanoparticles (AgNPs). (A) HRSEM image of AgNPs with 100,000X magnification. (B) EDX analysis of AgNPs. 


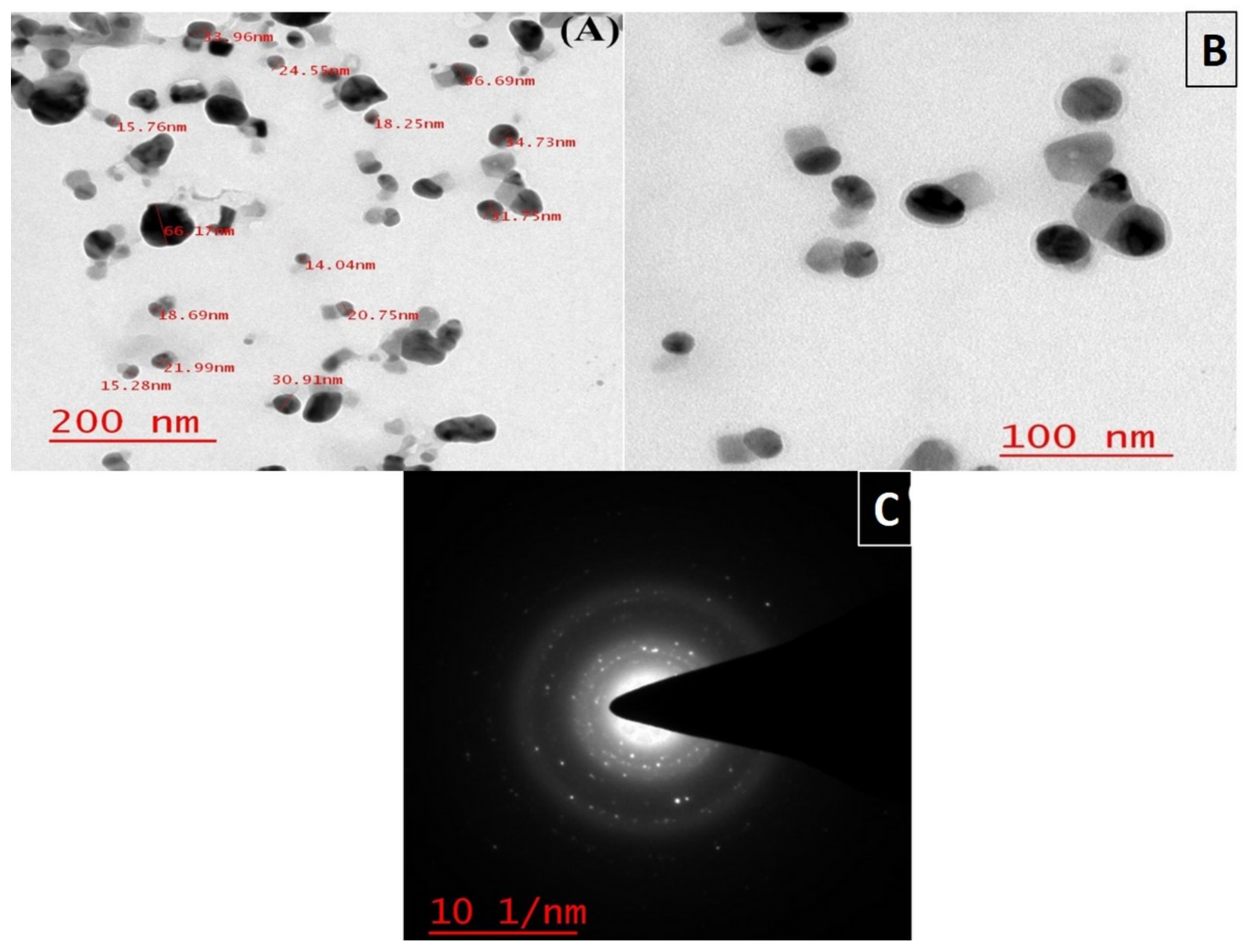

Figure 3

Characterization of the green synthesised silver nanoparticles (AgNPs). (A) and (B) HR-TEM images of AgNPs (C) Selected Area Electron Diffraction (SAED) pattern of AgNPs. 


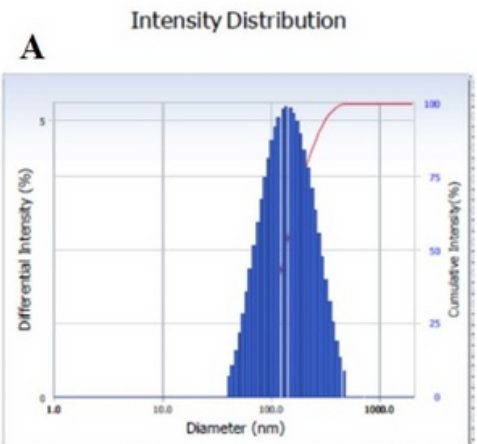

Cumulants Results
B

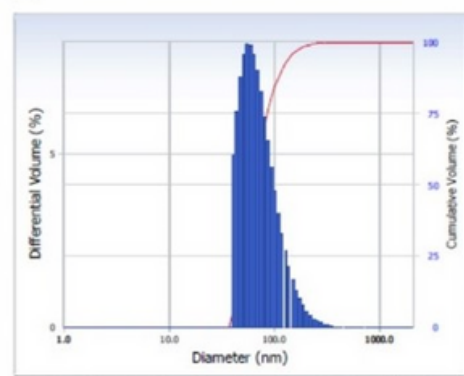

C Number Distribution

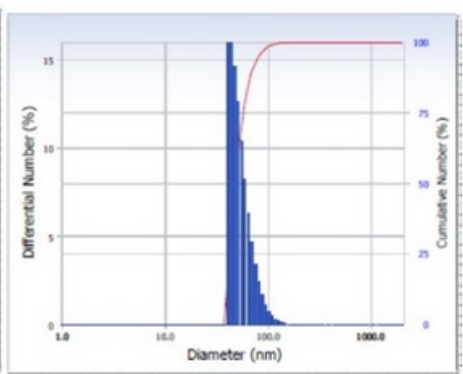

D

$\mathrm{LN}(\mathrm{G} 2(\mathrm{~T})-1)$ vS T

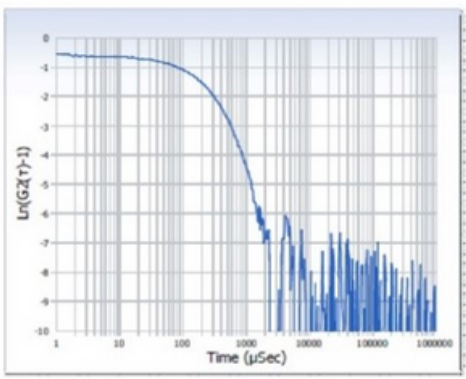

$\left({ }^{\circ} \mathrm{C}\right)$

Temperature : 25.0

Diluent Name : WATER

Refractive Index : : 1.3328

Viscosity : 0.8878

Scattering Intensity : 36096

Attenuator 1

: 0.12

(cps)

$(\%)$

Distribution Results (Contin) Intensity Distribution

Peak Diameter $(\mathrm{nm})$ Std. Dev.

$\begin{array}{ccc}\text { Peak } & \text { Diameter }(\mathrm{nm}) & \text { Std. Dev. } \\ 1 & 164.6 & 89.0 \\ 2 & 0.0 & 0.0 \\ 3 & 0.0 & 0.0 \\ 4 & 0.0 & 0.0 \\ 5 & 0.0 & 0.0\end{array}$

Average

164.6

89.0

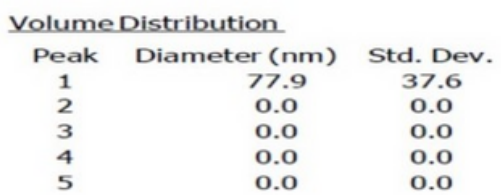

Average
Number Distribution

Peak Diameter $(\mathrm{nm})$ std. Dev.

$\begin{array}{ccc}\text { Peak } & \text { Diameter }(\mathrm{nm}) & \text { Std. Dev } \\ 1 & 55.1 & 15.9\end{array}$

$\begin{array}{ccc}1 & 55.1 & 15.9 \\ 2 & 0.0 & 0.0\end{array}$

$30.0 \quad 0.0$

$\begin{array}{lll}4 & 0.0 & 0.0 \\ 5 & 0.0 & 0.0\end{array}$

Average $\quad 55.1 \quad 15.9$

Figure 4

Particle size analysis of silver nanoparticles (AgNPs). (A) Intensity distribution (B) Volume distribution (C) Number distribution (D) Light scatter of AgNPs (E) Cumulative result 


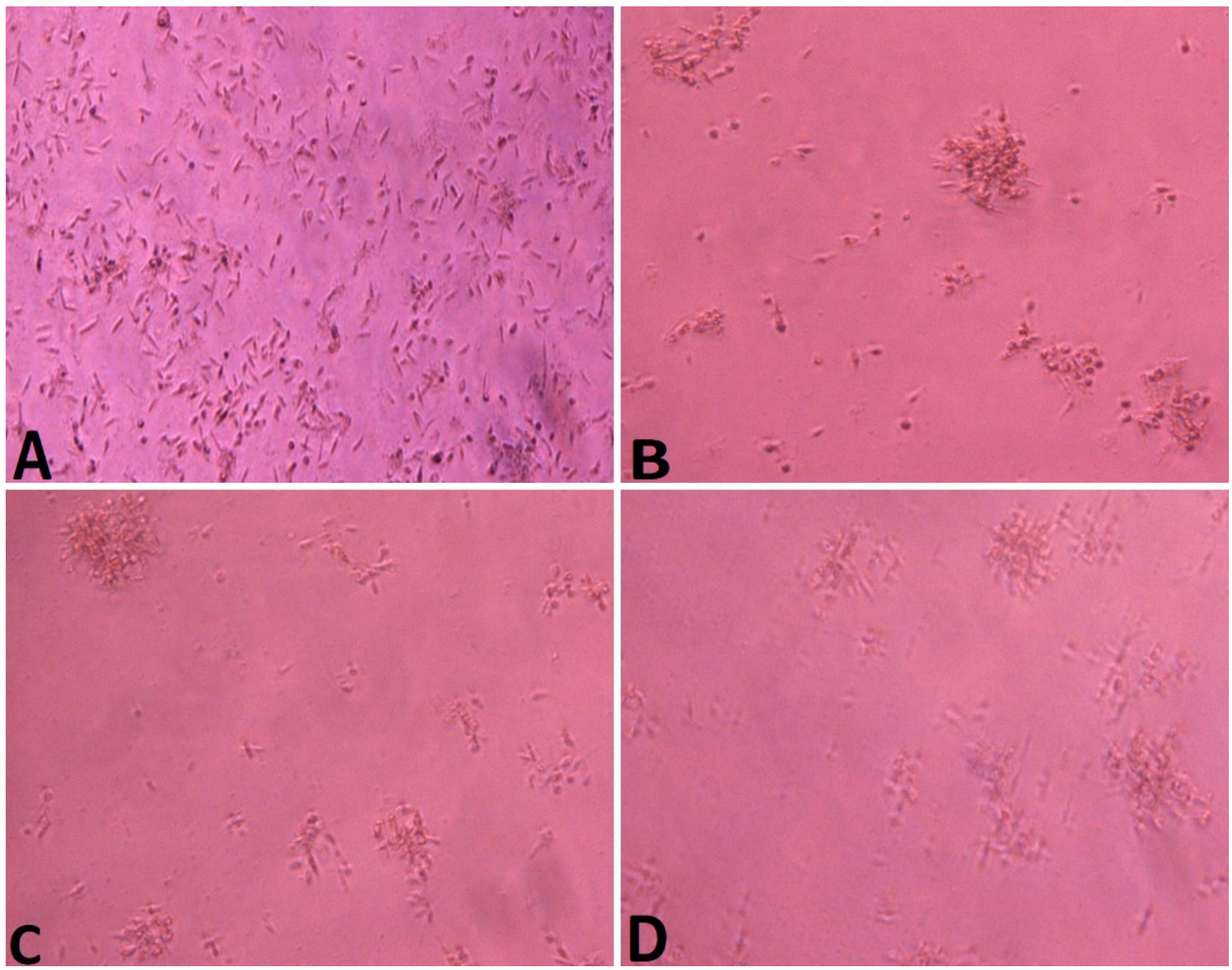

Figure 5

Anti-leishmanial activity of silver nanoparticles (AgNPs) on Leishmania donovani Promastigote in (A) control group (not exposed to silver nanoparticles [Ag-NPs] or aqeous extract [AE]) (B) AE treated group (C) AgNPs treated group and (D) Miltefosine treated group

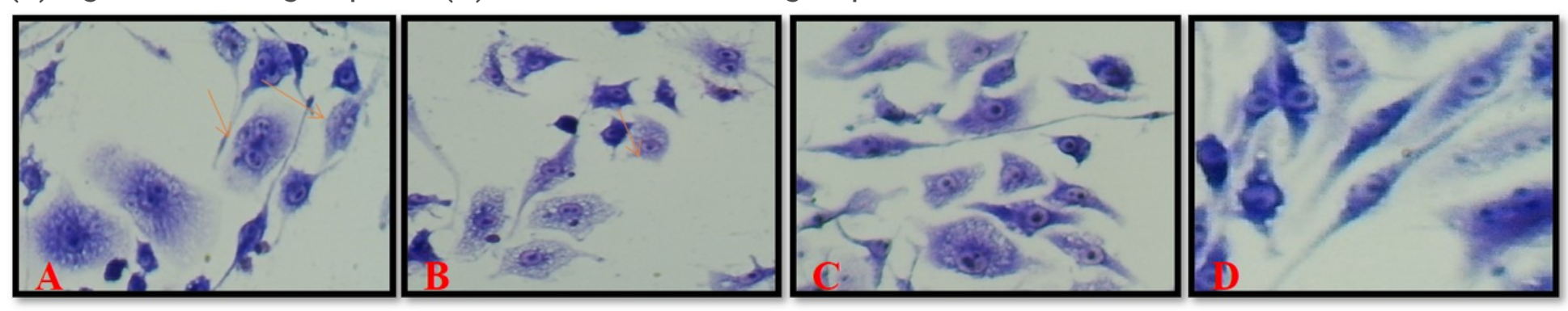

Figure 6 
Microscopic images of macrophages infected or not infected by parasites after being stained with Giemsa. Leishmania donovani promastigotes in (A) control group (not exposed to silver nanoparticles [Ag-NPs] or aqeous extract [AE]). (B) L. donovani promastigotes exposed to AE treated group and (C) AgNPs treated group infected macrophages, but their infectivity was very low compared with the control group and is comparable with (D) Miltefosine treated group.

Se

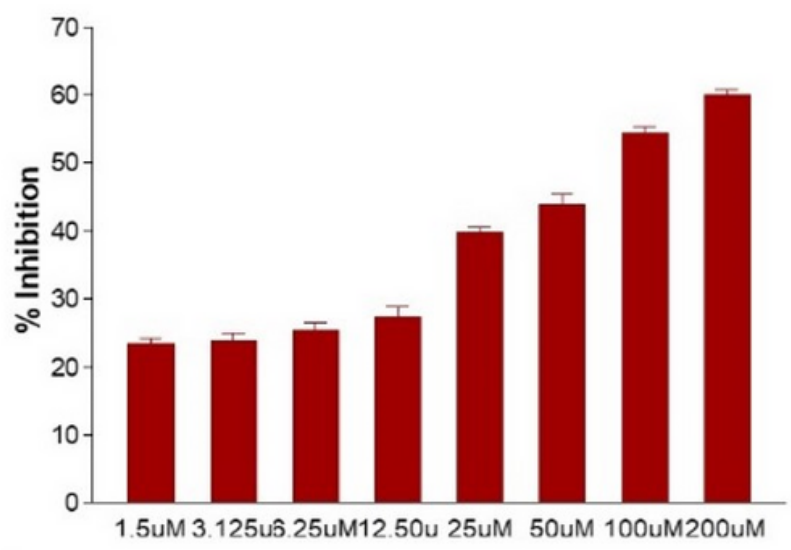

A

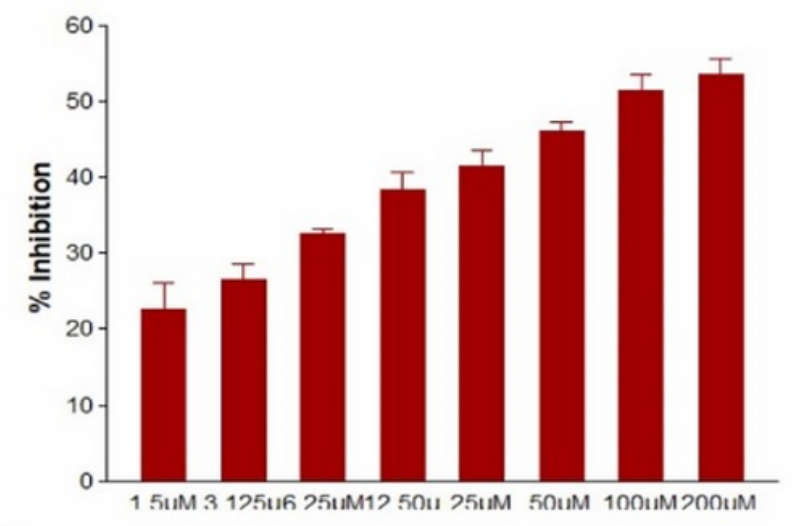

C
SG

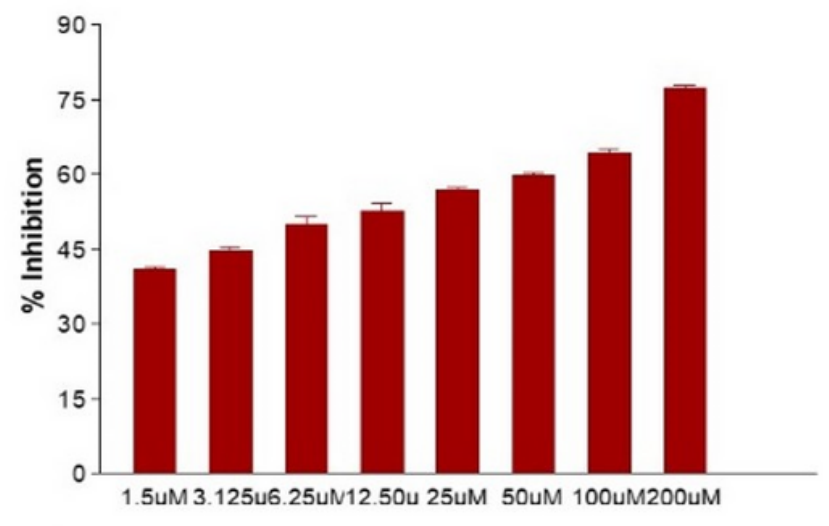

B

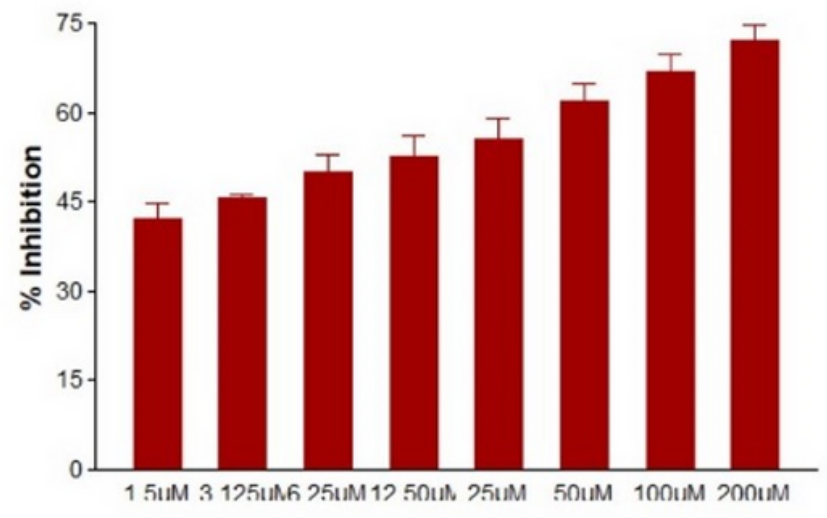

D

\section{Figure 7}

Percent cell viability on Leishmania donovani promastigotes exposed to (A) aqeous extract (AE) and (B) silver nanoparticles (AgNPs). Percent cell viability on Leishmania donovani amastigotes exposed to (C) AE and (D) AgNPs.

\section{Supplementary Files}

This is a list of supplementary files associated with this preprint. Click to download.

- GraphicalAbstract.png

- Supplementaryfile.docx 